\title{
Antibiotic Profile of Pseudomonas aeruginosa Isolated from Patients Visiting Grecian Super Specialty Hospital, Mohali, India
}

\author{
Manpreet Kaur $^{1 *}$ and Lovleen Pal ${ }^{2}$ \\ ${ }^{1}$ Department of Microbiology, Grecian Super Specialty Hospital, Sector 69, Mohali, India \\ ${ }^{2}$ Department of Anaesthesia, Max Hospital, Mohali, India \\ *Corresponding author
}

\section{A B S T R A C T}

\begin{abstract}
Keywords
Pseudomonas aeruginosa, clinical isolates, Resistant, Susceptible.

Article Info

Accepted:

20 December 2019

Available Online:

20 January 2020

Antimicrobial resistance especially in Pseudomonas spp. is an increasing problem across the world and therefore, its trend of antimicrobial resistance requires to be studied. The objective of current study was to determine the prevalence of Pseudomonas aeruginosa in various clinical samples collected during study and to analyze the antibiotic susceptibility patterns of various drugs against it. During the study, a total of $231 \mathrm{P}$. aeruginosa isolated from 870 clinical samples like branchoalveolar fluid (BAL), blood, endotracheal secretion, Foleys tip culture, pleurineral flurineid, pus, spurineturinem, sputum, swab curinelturinere, urine and wound. Antibiotic susceptibility testing were carried out according to the recommendations of Clinical Laboratory Standards Institute (CLSI) guidelines. The highest number of Pseudomonas infections was found in urine, followed by endotracheal, blood, BAL, spurineturinem, wound, pus, foley's tip, pleurineral flurineid, sputum and swab. Tobracef was the most active drug tested ( $82.25 \%$ susceptible), followed by pipercillin+tazobactam $(74.89 \%$ susceptible), cefoperazone+sulbactam $(67.10 \%$ susceptible), meropenem (64.94\% susceptible) and ceftazidime (60.17\% susceptible). From the present study, it appears that lower respiratory tract infection was the most common hospital acquired infection caused by multidrug resistant (MDR) $P$. aeruginosa. Moderate rates of drug resistance among $P$. aeruginosa isolates were observed against ceftazidime and meropenem. This pattern of resistance indicates probable overuse of broad-spectrum antibiotics without the having knowledge of sensitivity patterns of drugs. So there is a continuous need of conduction of surveillance programmes to recommend appropriate therapy to patients. Moreover, our data also demonstrated that Tobracef has enhanced in-vitro antibacterial activity and exhibited $17>\%$ superiority over meropenem. Therefore, it can be a better choice to treat the infections caused by multi-drug resistant $P$. aeruginosa.
\end{abstract}

\section{Introduction}

Pseudomonas is an aerobic, motile, opportunistic problematic Gram-negative bacterium and has been implicated in diverse healthcare associated infections (HCAIs) such as lower respiratory tract infection, urinary tract infection, skin and soft-tissue infections, in severe burns and in infections among immune compromised individuals (Tadvi et al., 2017; Siddiqua et al., 2018; Rao et al., 2017; Prasad et al., 2017). It is responsible for $10-15 \%$ of nosocomial infections across the world (Hancock and Knowles, 1998; Maniatis et al., 1997; Blanc et al., 1998). In critically ill patients, $P$. aeruginosa contributes $3 \%-15 \%$ of blood stream infections with high mortality rate of about $27 \%-48 \%$. In spite of recent advances in therapy, $P$. aeruginosa bacteremia remains 
fatal in more than $20 \%$ of cases. Over $50 \%$ of deaths happen within a few days of infection (Pragasam et al., 2018). It is the most common pathogen recovered from lower respiratory tract infection (LTRIs).

LRTI is one of the leading causes of morbidity and mortality worldwide (Alter et al., 2011). In addition, of total 3,941,000 deaths in the world, LRTI accounts for $34.60 \%$ deaths in the South-East Region (WHO, 2002). In developing countries, the situation is more complicated, and management is often difficult due to the problem associated with the identification of the etiological agents and the administration of an appropriate treatment in cases requiring antibiotic therapy (Khan et al., 2015).

Earlier studies have reported that $P$. aeruginosa to be most common isolate (35$59 \%$ ) from patients with LRTIs (Goel et al., 2009; Khan et al., 2015; Ozyilmaz et al., 2005; Gladstone et al., 2005; Imani et al., 2005). Infections with multidrug-resistant Pseudomonas are not only associated with considerable morbidity and mortality, but it also presents an economic burden as these are associated with high treatment costs and longer duration of hospital stay when compared to those associated with their drugsusceptible counterparts (Livermore, 2002; Mauldin et al., 2010).

The prime reason for morbidity, mortality and healthcare cost is the rapidly growing antibiotic resistance (Camell et al., 1993; Medina and Pieper, 2016). Pseudomonas exhibits a high level of intrinsic resistance to antimicrobial drugs and an ability to become even more drug resistant due to production of ESBL enzymes, AmpC hyper expression, repression or inactivation of oprD, over expression of efflux pumps and low permeability of its outer membrane (Aloush et al., 2006; Livermore, 2002; Livermore, 1984).
Besides, Pseudomonas species acquires class B metallo- $\beta$-lactamases (MBLs) which are another antibiotic resistance determinants and can be disseminated horizontally through transfer of mobile genetic elements coding for MBLs (Livermore, 2002).

Over the years, increases in the rate of antibiotic resistance to $P$. aeruginosa, in particular to beta-lactams, aminoglycosides, and fluoroquinolones, has been reported from many parts of the world (Jones et al., 2002). However, such problems are of great concern in developing countries particularly due to easy availability of antibiotics over the counter (Martinez, 2002).

A study by Gupta et al. (2016) from Aligarh, India reported an average $76.1 \%, 70.2 \%$ and 65-68\% of the isolated Pseudomonas species were resistant to fluoroquinolones, aminoglycosides and cephalosporins, respectively. Several other studies from India as well as Indian Council of Medical research (ICMR) also highlighted the resistance of Pseudomonas species against monotherapy of penicillins, cephalosporins, fluoroquinolones, tetracyclines and macrolides (Javiya et al., 2008; Walia et al., 2019; Kumari et al., 2019; Ramana and Chaudhury, 2012; Chaudhary and Payasi, 2013; Prakash et al., 2014). A decreased susceptibility rate of $P$. aeruginosa to $\beta$-lactams, carbapenems, quinolones and aminoglycosides has been reported in various countries (Khan and Faiz, 2016; Mansoor et al., 2009; Féria et al., 2002).

Therefore, there is a need to conduct surveillance studies of Pseudomonas for its resistance pattern. The present study was undertaken to find out the drug resistance and antibiotic susceptibility patterns in Pseudomonas species isolated from different clinical specimens of patients at a tertiary care hospital of India. 


\section{Materials and Methods}

\section{Sample processing}

Two hundred and thirty one samples collected aseptically from brancho-alveolar fluid (BAL), blood, endotracheal secretion, Foleys tip culture, pleurineral flurineid, pus, spurineturinem, sputum, swab curinelturinere, urine and wound were reported the presence of bacterial infection. The samples were inoculated on different media like Nutrient Agar, Blood Agar and MacConkey Agar for isolation as per the Standard Operative Procedure. $P$. aeruginosa were further identified by use of Indole, Citrate, Triple sugar iron (TSI), Catalase, Oxidase and physiologically by Urea hydrolysis and Mannitol motility (Koneman, 2006).

\section{Antibiotic susceptibility testing}

The antimicrobial susceptibility testing was performed by Kirby Bauer's disk diffusion method in accordance to the guidelines published by Clinical Laboratory Standards Institute (CLSI) (CLSI, 2018). Inoculum of 0.5 McFarland standards turbidity was prepared from isolated colony of pathogens selected from 18-24 hour agar plates. Within 15 minutes, a sterile cotton swab was dipped into the inoculum suspension. The swab was rotated several times and pressed firmly against the inside wall of the tube above the fluid level and inoculated on the dried surface of a Mueller-Hinton agar (MHA) plate by streaking the swab over it. For even distribution of inoculum, the swab was streaked two more times at $60^{\circ}$ over the agar surface. After 3-5 minutes, antibiotic discs were applied and pressed down to ensure complete contact with agar surface. The discs were distributed evenly to ensure a minimum distance of $24 \mathrm{~mm}$ from center to center. The plates are then inverted and incubated for 1618 hrs aerobically at $37^{\circ} \mathrm{C}$ within 15 minutes of disc application and zone of inhibition measured in millimetres and the results were interpreted as Sensitive, Intermediate and Resistant.

Paper disks impregnated with following antibiotics were used in the study: Tobracef (ceftazidime+tobramycin) $\quad(40 \quad \mu \mathrm{g})$, ceftazidime $(30 \mu \mathrm{g})$, cefoperazone +sulbactam $(105 \mu \mathrm{g})$, piperacillin+tazobactam $(110 \mu \mathrm{g})$ and meropenem $(10 \mu \mathrm{g})$. Discs of these drugs were purchased from Indian market on behalf of sponsor.

\section{Results and Discussion}

The increasing of infections caused by multidrug resistant bacteria has now become a major threat in medical world. Now-days, $P$. aeruginosa is considered a leading cause of gram negative bacterial infections especially in immuno-suppressed patients who need prolonged hospitalization (Arora et al., 2011).

A total 870 different clinical samples were collected from patients visiting Grecian Super Specialty Hospital, Mohali and processed for isolation of pathogenic bacteria. Eleven types of clinical samples included brancho-alveolar fluid (BAL), blood, endotracheal secretion, Foleys tip culture, pleurineral flurineid, pus, spurineturinem, sputum, swab curinelturinere, urine and wound.

A total of $231 P$. aeruginosa isolates were obtained from 870 clinical samples. The maximum $P$. aeruginosa was recovered from urine samples $(39.83 \%)$ followed by endotracheal section which contributed to $23.38 \%$, indicating urinary tract and endotracheal infections are most common hospital acquired infections. These results are in line with earlier study (Raman and Chaudhary, 2012). Each of blood, BAL and spurineturinem samples contributed to $5.63 \%$. Share of wound and pus sample was 5.19\% 
each. However, samples from foley's tip, pleurineral flurineid, sputum and swab curinelturinere contributed to $<5 \%$ (Table 1 ; Figure 1). Rajkumari et al. (2014) reported that the most common sample from which P.aeruginosa was recovered was from urine samples $(29.0 \%)$, followed by tracheal aspirates $(24.4 \%)$, pus/wound swabs $(20.0 \%)$, blood $(8.0 \%)$, bronchalveolar lavage $(8.0 \%)$, tissues $(1 \%)$, and sputum $(0.1 \%)$.Another study by Javiya et al. (2008) reported highest number of $P$. aeruginosa from urine followed by pus and sputum.

Fig.1 shows that the Prevalence percentage of clinical pathogens among different clinical samples. In the present study $82.25 \%$ of isolated multi-drug resistant (MDR) bacteria were observed to be susceptible to Tobracef which is consistent with previous studies (Chaudhary and Payasi, 2015). The higher susceptibility of Tobracef in $P$. aeruginosa results from enhanced level of drug uptake and higher outer membrane permeability (Chaudhary et al., 2014).This observation highlights the importance of Tobracef in MDR Gram-negative pathogens in causing LRTI.

The ureidopenicillin group represented by piperacillin+tazobactam appeared to be second most susceptible drugs exhibiting $74.89 \%$ susceptibility this result corroborate a previous study of Arumugam et al. (2018). Amongst third generation cephalosporins, ceftazidime is the drug used to treat Pseudomonal infections.In the current study, it showed $60.17 \%$ susceptibility to $P$. aeruginosa. However, over the past few years, Pseudomonal resistance to this drugs has increasingly been reported (Arora et al., 2011; Valenza et al., 2010).

Studies carried out by previous authors (Chitnis et al., 2003; Laura et al., 2000) in cephalosporins showed that the overall resistance to various generations of cephalosporins was high on MDR pathogens (i.e. resistance to at least three antibiotics) due to various mechanism including production of antibiotic inactivating enzyme. Pathogens maintain their MDR phenotype and spread throughout the community. Addition of betalactamase inhibitor (sulbactum) to cephalosporins monotherapy significantly reduced the percentage resistance and increased the percentage susceptibility against all the organisms. In the current study, cefoperazone+sulbactama (a combination of beta-lactam and beta-lactamase inhibitor) showed $67.10 \%$ susceptibility. Amongst the beta-lactam and beta-lactamase inhibitor group, cefoperazone+sulbactam showed resistance of $32.90 \%$. Increasing resistance of $P$. aeruginosa against $\beta$-lactamase inhibitor antibiotics may be due to excessive $\beta$ lactamase production and/or active efflux mechanism may also contribute to the full expression of $\beta$-lactam resistance in $P$. aeruginosa.Multi drug efflux pumps in the inner and outer membrane of $P$. aeruginosa may protect the bacterium from to $\beta$-lactam agents (Srikumar et al., 1997).

Carbepenems such as meropenem is often used as last choice for treatment of infection by Pseudomonas. In the current study showed a percentage susceptibility of $64.94 \%$ against meropenem. Supporting this observation, Arumugam et al. (2018) reported $73.6 \%$ susceptibility against meropenem. Another study by Javiya et al. (2008) reported $69.64 \%$ susceptibility against meropenem.

Currently the resistance towards this group of drugs is increasing. We observed the resistance of $35.06 \%$ for meropenems. The resistance to carbapenems, especially in MDR $P$. aeruginosa, results from reduced levels of drug accumulation or increased expression of pump efflux (Gupta et al., 2006; Kurokawa et al., 1999). 
Table.1 A profile of clinical samples used as a source of the pathogenic isolates

\begin{tabular}{|c|l|c|c|}
\hline Sr. No. & \multicolumn{1}{|c|}{ Specimen } & \% & No. \\
\hline 1 & BAL & 5.63 & 13 \\
\hline 2 & Blood & 5.63 & 13 \\
\hline 3 & Endotracheal & 23.38 & 54 \\
\hline 4 & Foley's tip culture & 0.87 & 2 \\
\hline 5 & Pleurineral flurineid & 4.76 & 11 \\
\hline 6 & Pus & 5.19 & 12 \\
\hline 7 & Spurineturinem & 5.63 & 13 \\
\hline 8 & Sputum & 3.46 & 8 \\
\hline 9 & Swab curinelturinere & 0.43 & 1 \\
\hline 10 & Urine & 39.83 & 92 \\
\hline 11 & Wound & 5.19 & 12 \\
\hline
\end{tabular}

Figure.1 Prevalence percentage of clinical pathogens among different clinical samples.

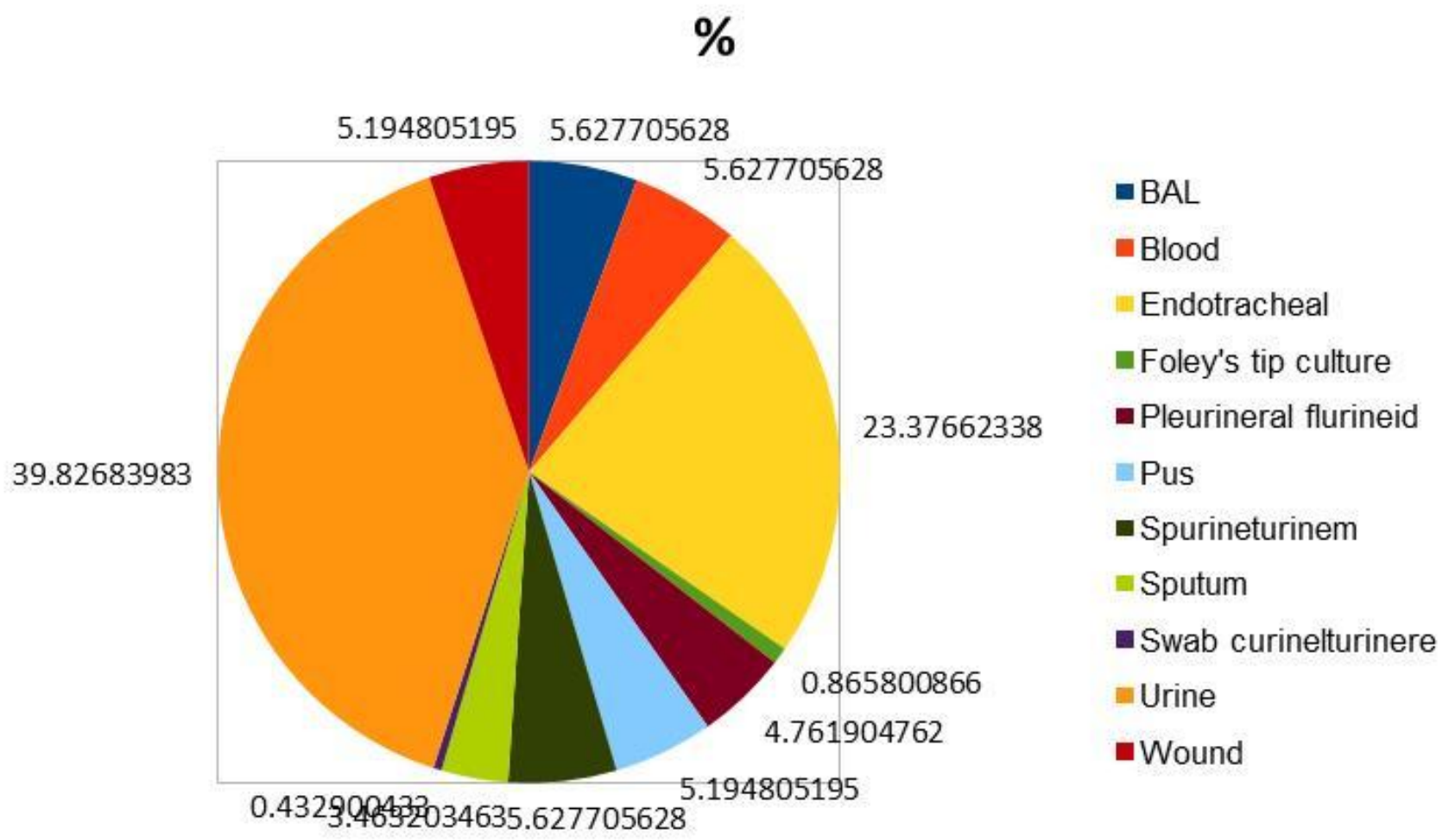


Figure.2 Susceptibility pattern of clinical isolates towards different antibacterial agents.

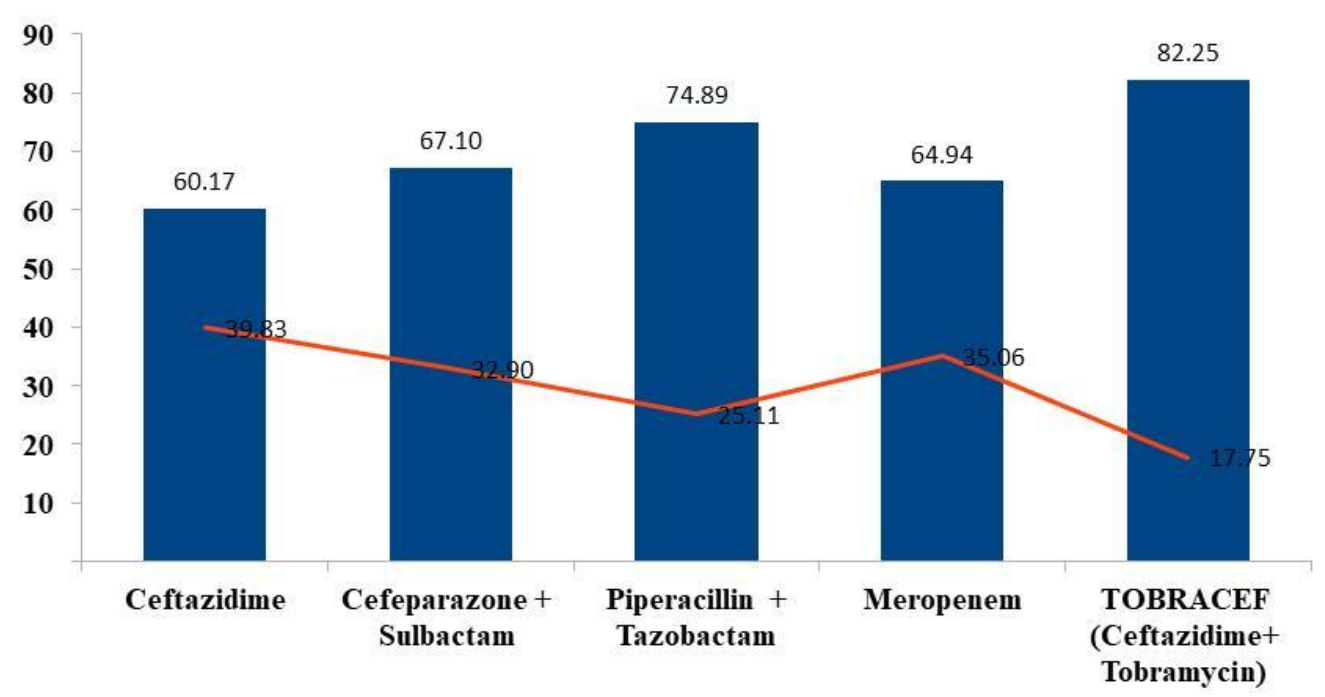

From the present study, it appears that lower respiratory tract infection was the most common hospital acquired infection caused by multidrug resistant $P$. aeruginosa. Moderate rates of drug resistance among $P$. aeruginosa isolates were observed against ceftazidime and meropenem. This pattern of resistance indicates probable overuse of broad-spectrum antibiotics without the having knowledge of sensitivity patterns of drugs. So there is a continuous need of conduction of surveillance programmes to recommend appropriate therapy to patients. Moreover, our data also demonstrated that Tobracef has enhanced in-vitro antibacterial activity and exhibited $17>\%$ superiority over meropenem. Therefore, it can be a better choice to treat the infections caused by multi-drug resistant (MDR) $P$. aeruginosa.

\section{References}

Alter SJ, Vidwan NK, Sobande PO, Omoloja A, Bennett JS. Common childhood bacterial infections. Curr Probl Pediatr Adolesc Health Care. 2011; 41: 256-83.

Aloush V, Navon-Venezia S, Seigman-Igra Y, Cabili S, Carmeli Y. Multidrug-resistant Pseudomonas aeruginosa: Risk factors and clinical impact. Antimicrob Agents Chemother, 2006; 50: 43-8.
Arumugam SN, Rudraradhya AC, Sadagopan S, Sukumaran S, Sambasivam G, Ramesh N. Analysis of susceptibility patterns of Pseudomonas aeruginosa and isolation, characterization of lytic bacteriophages targeting multi drug resistant Pseudomonas aeruginosa. Biomed Pharmacol J 2018; 11(2).

Arora D, Jindal N, Kumar R, Romit. Emerging antibiotic resistance in Pseudomonasa challenge. Int. J. Phar. Pharmaceut. Sci, 2011; 3: 82-84, 2011.

Blanc DS, Petignat C, Janin B, Bille J, Francioli $\mathrm{P}$. Frequency and molecular diversity of Pseudomonas aeruginosa upon admission and during hospitalization: a prospective epidemiologic study. Clin Microbiol Infect 1998; 4: 242-247.

Clinical and Laboratory Standards Institute. Performance standards for antimicrobial susceptibility testing; twenty-third informational supplement. CLSI document M100-S28. 2018; Wayne, PA 19387 USA.

Chaudhary M, Payasi A. Rising Antimicrobial Resistance of Pseudomonas aeruginosa Isolated from

Clinical Specimens in India. J Proteomics Bioinform, 2013; 6(1): 005-009.

Camell Y, Troillet N, Eliopoulos GM, Samore MH. emergence of antibiotic-resistant Pseudomonas aeruginosa: comparison of risks associated with different 
antipseudomonal agents. Antimicrob Agents Chemother, 1999; 43: 1379-1382.

Chitnis SV, Chitnis V, Sharma N, Chitnis DS. Current status of drug resistance among gram negative bacilli isolated from admitted cases in a tertiary care centre. J Assoc Physicians India, 2003; 51:28-32

Chaudhary M, Payasi A. Comparative in vitro activity of Tobracef against Gram negative isolates. Int J Rec Sci Res, 2015; 6: 41024106.

Chaudhary M, Bansal R, Payasi A. Overcoming Pseudomonas aeruginosa Resistance Caused by Glycocalyx with Tobracef. J Microb Biochem Technol, 2014; 6(4): 207-211.

Féria C, Ferreira E, Correia JD, Gonçalves J, Caniça M. Patterns and mechanisms of resistance to beta-lactams and beta-lactamase inhibitors in uropathogenic Escherichia coli isolated from dogs in Portugal. J Antimicrob Chemother, 2002; 49: 77-85.

Goel N, Chaudhary U, Aggarwal R, Bala K. Antibiotic sensitivity pattern of Gram negative bacilli isolated from the lower respiratory tract of ventilated patients in the Intensive care unit. Indian J Crit Care Med, 2009;13:148-151.

Gupta R, Malik A, Rizvi M, Ahmed S M. Incidence of multidrug-resistant pseudomonas spp. in ICU patients with special reference to ESBL, AMPC, MBL and biofilm production.J Global Infect Dis, 2016;8:25-31.

Gupta E, Mohanty S, Sood S, Dhawan B, Das BK, Kapil A. Emerging resistance to Carbapenems in a tertiary care hospital in North India. Indian J Med Res 2006;124:958.

Gladstone P, Rajendran P, Brahmadathan KN. Incidence of carbapenem resistant nonfermenting gram negative bacilli from patients with respiratory infections in the intensive care units. Indian J Med Microbiol, 2005; 23(3):189-91.

Hancock REW, Knowles D. Are we approaching the end of the antibiotic era? Editorial overview. Curr Opinion Microbiol, 1998; I: 493-494.

Imani R, Rouhi H, Ganji F. Prevalence of antibiotic resistance among bacteria isolates of lower respiratory tract infection in COPD
Shahrekord, Iran, 2005. Pak J Med Sci, 2007; 23(3): 438-40.

Javiya VA, Ghatak SB, Patel KR, Patel JA. Antibiotic susceptibility patterns of Pseudomonas aeruginosa at a tertiary care hospital in Gujarat, India.Indian J Pharmacol 2008; 40:230-234

Jones Rn, Kirby JT, Beach ML, Biedenbach DJ, Pfaller MA. Geographic variations in activity of broad-spectrum beta-lactams against Pseudomonas aeruginosa: summary of the worldwide SenTRY Antimicrobial Surveillance Program (1997-2000). Diagn Microbiol Infect Dis, 2002; 43: 239-243.

Kumari M, Surbhi Khurana, Nidhi Bhardwaj, Rajesh Malhotra, and Purva Mathur. Pathogen burden \& associated antibiogram of Pseudomonas spp. In a tertiary care hospital of India. Indian J Med Res, 2019; 149(2): 295-298.

Koneman Koneman's., "Color Atlas and textbook of diagnostic Microbiology". The Non fermantative Gram-Negative Bacilli 6 (2006): 303-391.

Kurokawa H, Yagi T, Shibata N, Shibayama K, Arakawa Y. Worldwide proliferation of Carbapenem resistant gram negative bacteria. Lancet, 1999;354:955.

Khan MA, Faiz A. Antimicrobial resistance patterns of Pseudomonas aeruginosa in tertiary care hospitals of Makkah and Jeddah. Ann Saudi Med, 2016; 36: 23-28.

Khan S, Priti S, Ankit S. Bacteria Etiological Agents Causing Lower Respiratory Tract Infections and Their Resistance Patterns. Iran Biomed J, 2015; 19: 240-246.

Khan JA, Iqbal Z, Rahman SU, Farzana K, Khan A. Report: Prevalence and resistance pattern of Pseudomonas aeruginosa against various antibiotics. Pak J Pharm Sci 2008; 21 : 3115.

Livermore DM. Multiple mechanisms of antimicrobial resistance in Pseudomonas aeruginosa: Our worst nightmare? Clin Infect Dis 2002;34:634-40.

Livermore DM, Penicillin-binding proteins, porins and outer-membrane permeability of carbenicillin-resistant and -susceptible strains of Pseudomonas aeruginosa. J Med Microbiol, 1984; 18: 261-270. 
Laura V, Pezzella C, Tosini F, Visca P, Petrucca A, Carattoli A. Multiple antibiotic resistance mediated by structurally related IncL/M plasmids carrying an ESL gene and a Class 1 Integron. Antimicrob Agent Chemother, 2000; 44: 2911-14.

Mansoor T, Musani MA, Khalid G, Kamal M. Pseudomonas aeruginosa in chronic suppurative otitis media: sensitivity spectrum against various antibiotics in Karachi. J Ayub Med Coll Abbottabad 2009; 21: $120-123$.

Martinez JL, Baquero F. Interactions among strategies associated with bacterial infection: Pathogenicity, epidemicity and antibiotic resistance. Clin Microbiol Rev, 2002;15:647-79.

Median E, Pieper DH. Tackling Threats and Future Problems of Multidrug-Resistant Bacteria. Curr Top Microbiol Immunol, 2016; 398:3-33.

Mauldin PD, Salgado CD, Hansen IS, Durup DT, Bosso JA. Attributable hospital cost and length of stay associated with health careassociated infections caused by antibioticresistant Gram-negative bacteria. Antimicrob Agents Chemother, 2010; 54 :109-15

Maniatis AN, Trougakos IP, Katsanis G et al. Changing patterns of bacterial nosocomial infections: a nine year study in a general hospital. Chemother, 1997; 43: 69-76.

Ozyilmaz E, Akan OA, Gulhan M, Ahmad K, Nagatake T. Major bacteria of community- acquired respiratory tract infections in Turkey. Jpn J Infect Dis, 2005; 58(1):50-2.

Prasad RR, Shree V, Kumar R, Kala K, Kumar P. Prevalence and antibiotic sensitivity of Pseudomonas aeruginosa isolated from CSOM in NMCH, Patna, India. Int $\mathbf{J}$ Curr Microbiol App Sci, 2017; 6 : 2912-6.

Prakash V, Prem Prakash Mishra, H. K. Premi, Apoorva Walia, Shikha Dhawan, Abhishek Kumar. Increasing incidence of multidrug resistant Pseudomonas aeruginosa in inpatients of a tertiary care hospital. Int J Res Med Sci. 2014; 2(4):1302-1306.

Pragasam AK, Veeraraghavan B, Nalini E, Anandan S, Kaye KS. An update on antimicrobial resistance and the role of newer antimicrobial agents for Pseudomonas aeruginosa. Indian J Med Microbiol [serial online] 2018 [cited 2019 Sep 30 ];36:303316.

Ramana BV, Chaudhury A. Antibiotic resistance pattern of Pseudomonas aeruginosa isolated from healthcare associated infections at a tertiary care hospital. J Sci Soc 2012;39:7880

Rao S, Subbarayudu S. Surveillance of Pseudomonas in COPD patients in a tertiary care hospital. Int J Res Med Sci, 2017; 3 : 1209-12.

Rajkumari N, John NV, Mathur P, Misra MC. Antimicrobial resistance in Pseudomonas sp. causing infections in trauma patients: A 6 year experience from a south asian country. $\mathbf{J}$ Global Infect Dis, 2014;6:182-5.

Srikumar R, Li XZ, Poole K. Inner membrane efflux components are responsible for $\beta$ lactam specificity of multi drug efflux pumps in Pseudomonas aeruginosa. J Bacteriol, 1997; 179: 7875-81

Siddiqua M, Alam AN, Akter S, Ferdousi RS. Antibiotic resistance pattern in Pseudomonas aeruginosa isolated from a private medical college hospital. KYAMC J, 2018; 9 : 16-9.

Tadvi J, Javadekar TB, Bhavsar R, Garala N. Prevalence \& antibiogram of Pseudomonas aeruginosa at SSG Hospital, Baroda, Gujarat, India. J Res Med Dent Sci, 2017; 3 : 204-7.

Valenza G, Radike K, Schoen C, Horn S, Oesterlein A, Frosch M, Abele-Horn M, Hebestreit H. Resistance to tobramycin and colistin in isolates of Pseudomonas aeruginosa from chronically colonized patients with cystic fibrosis under antimicrobial treatment. Scand J Infect Dis, 2010; 42: 885-9

Walia K, Jayaprakasam Madhumathi, Balaji Veeraraghavan, Arunaloke Chakrabarti, Arti Kapil,

Ray P, Singh H, Sistla S, Ohri VC. Establishing Antimicrobial Resistance Surveillance \& Research Network in India: Journey so far. Indian J Med Res, 2019;149 : 164-179.

World Health organization. Leading causes of deaths in the SEA Regionand the World. Health situation in the South- East Asia Region 1998- 2000. New Delhi. India: WHO; 2002. 
How to cite this article:

Manpreet Kaur and Lovleen Pal. 2020. Antibiotic Profile Of Pseudomonas aeruginosa Isolated From Patients Visiting Grecian Super Specialty Hospital, Mohali, India. Int.J.Curr.Microbiol.App.Sci. 9(01): 2588-2596. doi: https://doi.org/10.20546/ijcmas.2020.901.293 